\title{
An extension of the transpired skin-friction equation to compressible turbulent boundary layers
}

\author{
Atila P. Silva-Freire ${ }^{\dagger}$ \\ Engineering Department, Cambridge University, Trumpington Street, Cambridge CB2 IPZ, U.K.
}

(Received 4 December 1987 and in final form 29 February 1988)

\section{INTRODUCTION}

THE INJECTION or suction of fluid into the turbulent boundary layer has proved over the years to be an effective means of controlling the properties of fluid flowing over a surface. The result of this injection or suction of fluid is to modify the velocity and temperature distributions through the boundary layer so that the drag and the heat transfer are either reduced or increased. Most of the studies on this topic, however, deal with incompressible flow.

The majority of the results on supersonic flow comes from the work of Squire and his students at Cambridge University [1-5]. These results are given for Mach numbers up to 3.6 at various injection rates. Full tables of measured profiles are presented and expressions for the law of the wall and the law of wake are proposed. As for the incompressible case, the law of the wall is obtained by straightforward application of the mixing-length theory [1]. Unfortunately, in those works the skin-friction coefficients are evaluated by means of the momentum-integral equation which tends to be very inaccurate, thus definitive checks on the proposed law of the wall are impossible.

In a previous paper [6], the present author has proposed a skin-friction equation for transpired incompressible turbulent boundary layers. This equation is much less sensitive than the momentum-integral equation to small variations in the flow parameters so providing much more reliable results. The aim of this work is to extend this skin-friction equation to compressible flow.

The approach of transforming a compressible turbulent boundary layer into a corresponding incompressible flow has been pursued by several authors in the past with reasonable success. The underlying idea is to reduce the complex system of partial differential equations which governs the motion of a compressible flow into a simpler system, such as the system of equations for an incompressible flow. Solutions of the simpler system can then be transformed back to predict the behaviour of the solutions of the complex one. This procedure, however, still presents the difficult problem of selecting the right transformation parameters. An alternative approach is to use the concept of generalized velocity. Using the assumption that the mixing length is proportional to the wall distance and that the shear stress in the fluid is constant and equal to its value at the wall, Van Driest [7] solved the equations of motion and obtained, for an adiabatic flow

$$
\frac{u_{\infty}}{\xi u_{\mathrm{r}}}\left(\arcsin \xi-\arcsin \frac{\xi u}{u_{\infty}}\right)=-\frac{1}{K} \ln \frac{y}{\delta}
$$

where

$$
\xi^{2}=(\gamma-1) M^{2} /\left(2+(\gamma-1) M^{2}\right) .
$$

Comparison of equation (1) with the well-known $\log$ arithmic law of the wall for incompressible flow shows that

† Present address: Programa de Engenharia Mecanica (COPPE/UFRJ), Universidade Federal do Rio de Janeiro, Brazil CEP 21945. the compressibility effects can be accounted for if the incompressible velocity profile is replaced by the generalized velocity, $u^{*}$, defined by

$$
u^{*}=\frac{u_{\infty}}{\xi} \arcsin \left(\frac{\xi u}{u_{\infty}}\right) .
$$

The above equation is normally referred to as the Van Driest transformation. Of course, in the limit as $M \rightarrow 0$ equation (1) reduces to the incompressible flow case. The wake function does not appear in equation (1) due to the hypothesis that the mixing length is given by $l=K y$ throughout the boundary layer. However, Maise and McDonald [8] have shown that solution (1) can be extended to a boundary layer with a finite wake if one writes

$$
u_{\infty}^{*}-u^{*}=u_{\tau}\left(-\frac{1}{K} \ln \frac{y}{\delta}+\frac{\pi}{K}(2-w(y / \delta))\right)
$$

where $w(y / \delta$.) is Coles' wake function.

Squire [1] used the same procedure as Van Driest to derive a law of the wall for transpired compressible turbulent boundary layers; but the result is in the form of an elliptic integral and so cannot be integrated exactly. This feature of his solution hampered any attempt by him of obtaining a skin-friction equation. For this reason, it was decided to apply Van Driest's transformation directly to the expressions derived in ref. [6] to obtain a simple set of expressions which yield a skin-friction equation. This procedure, although not theoretically rigorous, is expected, together with adequate modifications in the incompressible results, to give good results.

\section{THE MOMENTUM-INTEGRAL EQUATION}

Values of the skin friction are normally evaluated in the literature by means of the momentum-integral equation which reads

$$
\frac{C_{\mathrm{f}}}{2}=\frac{\mathrm{d} \theta}{\mathrm{d} x}-F-\frac{\theta}{\rho_{\infty}^{2} u_{\infty}^{2}} \frac{\mathrm{d} p}{\mathrm{~d} x}\left(2+H-M_{\infty}^{2}\right)
$$

where $F$ is the injection rate $\left(=\rho_{\mathrm{w}} V_{\mathrm{w}} / \rho_{\infty} u_{\infty}\right), H$ the boundary layer shape parameter and $\theta$ the momentum thickness.

The difficulty in using equation (5) lies in the fact that $C_{f}$ is normally much smaller than the individual terms on the right-hand side and so is the result of a small difference between large numbers. This makes equation (5) strongly dependent on the accuracy of the experimental data. Unfortunately, this difficulty is not easily rectified due to the scatter in the experimental data. For example, most of the porous surfaces which are commercially available give an accuracy of only $\pm 7 \%$ in $F$. This causes an unavoidable variation in the injection velocity along the surface which greatly affects the results yielded by equation (5). This problem is further aggravated by the magnification of the scatter in the measurements by the differentiation [2]. In fact it is possible that the errors in $\mathrm{d} \theta / \mathrm{d} x$, in $F$ and in the pressure-gradient term are greater than 0.0002 . Consequently, equation (5) is subject to large random errors. 


\section{NOMENCLATURE}

$\begin{array}{ll}A & \text { constant in law of the wall } \\ C_{\mathrm{f}} & \text { skin-friction coefficient } \\ C_{\mathrm{fo}} & \text { skin-friction coefficient for unblown case } \\ F & \text { injection rate, } \rho_{\mathrm{w}} v_{\mathrm{w}} / \rho_{\infty} u_{\infty} \\ H & \text { boundary layer shape parameter } \\ K & \text { Von Karman's constant } \\ M & \text { Mach number } \\ p & \text { pressure } \\ R & \text { Reynolds number } \\ u & \text { tangential velocity } \\ u_{\mathrm{r}} & \text { friction velocity } \\ u_{\mathrm{e}} & u_{\infty}(\text { arcsin } \xi) / \xi \\ u^{*} & \text { generalized velocity } \\ v & \text { normal velocity } \\ x, y & \text { Cartesian coordinates } \\ w(y) & \text { Coles' function. }\end{array}$

\section{THE SKIN-FRICTION EQUATION FOR COMPRESSIBLE TRANSPIRED TURBULENT BOUNDARY LAYERS}

In this section equation (4) is extended to the case with transpiration. This is done by letting the right-hand side of this equation account for the blowing effects, that is, by replacing the logarithmic defect layer solution by a bilogarithmic defect layer solution such as that derived in ref. [6]. Note, however, that now, due to the compressibility, the thermodynamic properties of the flow change across the boundary layer and hence parameters such as Mach number, Reynolds number, and the normal velocity, need to be suitably defined. In this work, $M$ is the external Mach number, $R$ the Reynolds number based on properties evaluated at the wall temperature, the density used in the definition of the friction velocity is evaluated at the wall condition and a characteristic normal velocity, $v_{\text {wo }}$, is defined in the overlap region.

The defect layer solution for a transpired incompressible turbulent boundary layer is shown in ref. [6] to be given by

$$
\begin{aligned}
u_{\infty}-u= & u_{\tau}\left(\frac{-1}{K} \ln \frac{y}{\delta}+\frac{\pi}{K}(2-w(y / \delta))\right) \\
& +v_{\mathrm{w}}\left(\frac{-1}{4 K^{2}} \ln ^{2} \frac{y}{\delta}-\left(\frac{1}{2 K^{2}} \ln \frac{\delta}{\delta}+\frac{A}{2 K}\right) \ln \frac{y}{\delta}\right. \\
& \left.+\frac{\tilde{\pi}}{K^{2}}(2-w(y / \delta))\right)+\ldots=f\left(y, u_{\tau}, v_{\mathrm{w}}, \delta, v_{\mathrm{w}}\right) .
\end{aligned}
$$

Hence, it follows from equation (3) that the defect layer solution for a compressible transpired flow is

$$
u_{*}^{*}-u^{*}=f\left(y, u_{\tau}, v_{\text {wo }}, \delta, v_{\mathrm{w}}\right) \text {. }
$$

Parameters $A, \pi$ and $\tilde{\pi}$ are considered here to be identical to those defined in ref. [6]. The parameter $v_{w}$ in equation (6) is replaced by $v_{\text {wo }}$ in equation (7) because in a compressible flow the normal velocity varies across the boundary layer to order unity and so a reference velocity needs to be suitably chosen. Since solution (7) must match the wall layer solution, it is assumed that

$v_{\text {wo }}=$ characteristic normal velocity in the overlap region.

Matching arguments similar to those in ref. [6] applied to solution (7) and to the wall layer solution yield the skinfriction equation for compressible transpired turbulent boundary layers as

$$
\begin{aligned}
u_{\mathrm{\rho}}=\frac{u_{\tau}}{K} \ln \frac{\delta}{\delta}+v_{\mathrm{wo}} \frac{1}{4 K^{2}} \ln ^{2} \frac{\delta}{\delta}+ & u_{\mathrm{q}} A+\frac{v_{\mathrm{wo}}}{2 K} A \ln \frac{\delta}{\delta} \\
& +u_{\mathrm{s}} \frac{2 \pi}{K}+v_{\mathrm{wo}}\left(\frac{A^{2}}{4}+\frac{2 \tilde{\pi}}{K}\right)
\end{aligned}
$$

Greek symbols

$\gamma \quad$ ratio of specific heats

$\delta$ boundary layer thickness

$\delta \quad$ wall layer thickness

$\theta$ momentum thickness

$v \quad$ kinematic viscosity

$\xi^{2} \quad(\gamma-1) M^{2} /\left(2+(\gamma-1) M^{2}\right)$

$\pi \quad$ wake profile parameter

$\tilde{\pi} \quad$ wake profile parameter due to the injection

$\rho$ density.

\section{Subscripts \\ w wall condition \\ wo overlap region condition \\ $\infty \quad$ free-stream condition.}

where, $u_{\mathrm{e}}=u_{\infty}(\arcsin \xi) / \xi$ and the parameters are the same as in the incompressible case, that is

$$
\begin{gathered}
\pi=0.55 \\
\tilde{\pi}=-1.95 \ln \left(v_{\mathrm{wo}} / u_{\infty}\right)-3.1 \\
A=5.0-512\left(v_{\mathrm{wo}} / u_{\infty}\right) .
\end{gathered}
$$

The solution of the system defined by equations (8)-(11) determines $C_{\mathrm{f}}$ for given values of $M, u_{\infty}, \delta$ and $v_{\mathrm{wo}}$. The characteristic normal velocity in the overlap region, $v_{\mathrm{wo}}$, is determined assuming that the matching between the wall and defect regions is performed at $y / \delta=0.1$. Therefore

$$
v_{\text {wo }}=v(0.1) \text {. }
$$

Values of $v_{\text {wo }}$ based on conditions at the wall, and on conditions at the edge of the boundary layer, were also tested on equation (8). These conditions give results which are clearly inconsistent. Predictions of $C_{\ell}$ using equation (8) should then be compared to experimental results. However, due to the difficulty in obtaining reliable experimental values for the skin-friction velocity, it is important to describe the criterion adopted in this work to select the experimental data. The method used was in fact that proposed by Squire [1]. Values of $C_{f}$ obtained from the experiments of Squire [2] and of Jeromin [5] were plotted in the form $C_{f} / C_{\mathrm{r}}$ against $2 F / C_{\mathrm{f}}$, where $C_{m}$ is the skin-friction coefficient for the unblown case and $F$ the injection rate. Next these points were enclosed by scatter bands based on the experimental accuracy. The mean of these bands was then considered to be the actual variation of $C_{t} / C_{\mathrm{f}}$. The appropriate values of $C_{\mathrm{F}}$ were then taken from Fig. 1 for given values of $F$ and $C_{f 0}$.

The experimental details are summarized in Table 1. Skinfriction values predicted by equation (8) are plotted in Figs.



$2 F / C_{\text {fo }}$

FIG. 1. Mean values of $C_{\mathrm{f}}$. 
Table 1. Flow conditions according to refs. [2, 5]

\begin{tabular}{rlll}
\hline$F C$ & \multicolumn{1}{c}{$F$} & $M$ & $R / m \times 10^{-7}$ \\
\hline 1 & 0.0 & 1.80 & 1.100 \\
2 & 0.0013 & 1.80 & 1.100 \\
3 & 0.0025 & 1.80 & 1.100 \\
4 & 0.0031 & 1.80 & 1.100 \\
5 & 0.0 & 2.50 & 0.659 \\
6 & 0.0013 & 2.50 & 0.659 \\
7 & 0.0024 & 2.50 & 0.659 \\
8 & 0.0036 & 2.50 & 0.659 \\
9 & 0.0 & 3.60 & 0.530 \\
10 & 0.00065 & 3.60 & 0.530 \\
11 & 0.0012 & 3.60 & 0.530 \\
12 & 0.0021 & 3.60 & 0.530 \\
\hline
\end{tabular}

2(a)-(c) against values of $R_{\theta}, \theta$ the momentum thickness, for $M=1.8,2.5$ and 3.6. These figures also show values of $C_{r}$ obtained from Squire [2] and from Fig. 1. The overall agreement for a Mach number of 3.6 is very good. For Mach numbers of 1.8 and 2.5 , the results obtained through equation (8) and Fig. I agree quite well. Equation (5), however, gave results which seem to be higher than the actual values of $C_{f}$. One of the interesting features of the analysis

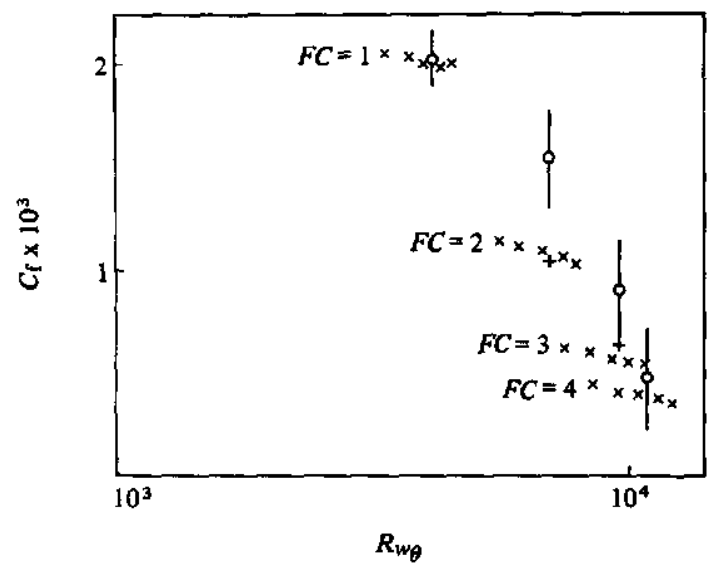

FIG. 2(a). Predictions of $C_{f}$ for $M=1.8: \times$, equation (8); $O$, Squire's results; + , Fig. 1 .



FIG. 2(b). Predictions of $C_{\mathrm{f}}$ for $M=2.5: \times$, equation (8); 0 , Squire's results; +, Fig. 1.

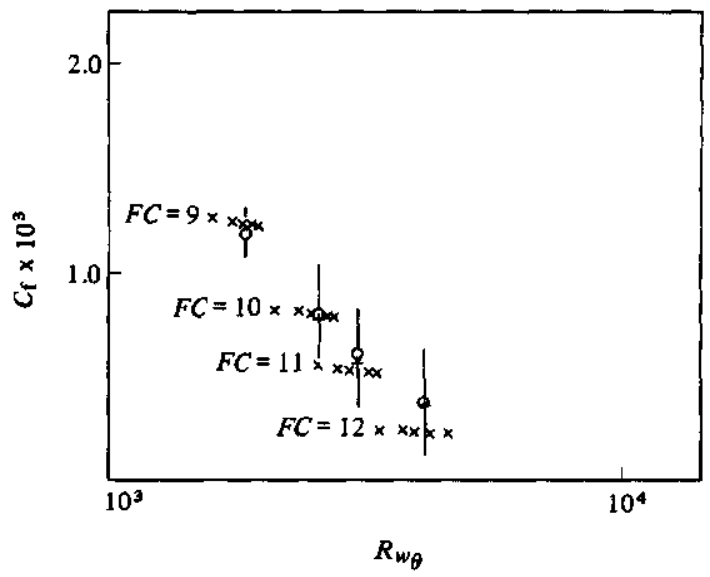

FIG. 2(c). Predictions of $C_{\mathrm{f}}$ for $M=3.6: \times$, equation (8); $O$, Squire's results; + , Fig. 1 .

carried out so far is that equation (8) has been derived using only physical arguments and the results of ref. [6]. Thus none of the parameters in equation (8) have been determined by a direct analysis of the experimental data for compressible flow. This yields an independent method which can be used to check the results given by equation (5). The velocity profiles were obtained substituting the predicted values of $C_{\mathrm{f}}$ into equation ( 7$)$. The results are shown in Figs. 3(a)-(c). For the lowest Mach number the theoretical predictions are reasonable. Unfortunately as the Mach number increases the agreement becomes systematically poorer, in particular for

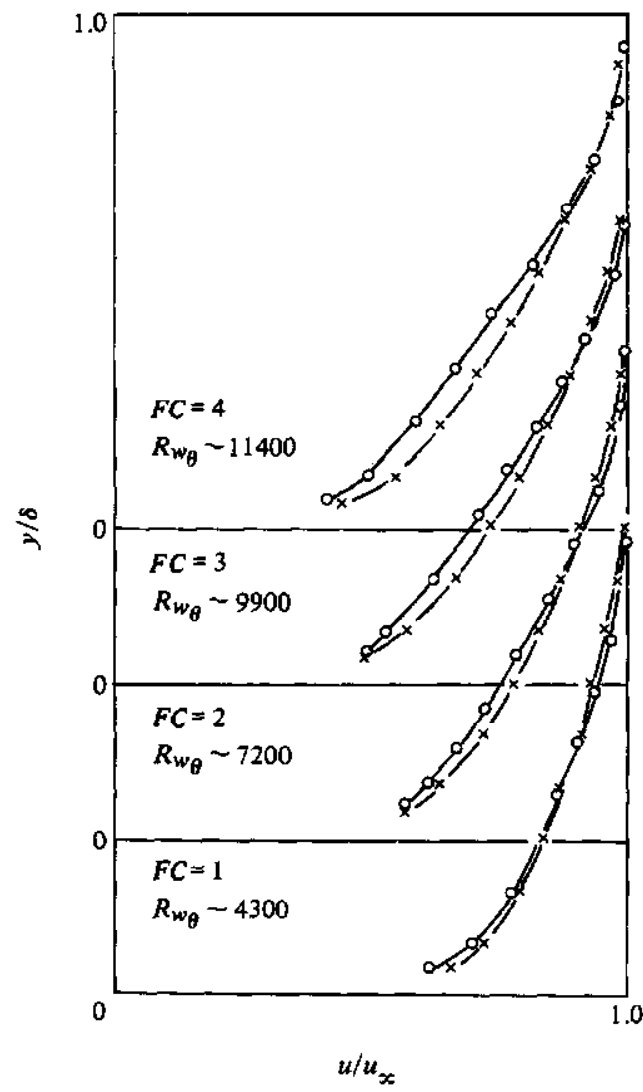

FIG. 3(a). Velocity profiles for $M=1.8: \times$, equation (7); $O$, experimental results. 


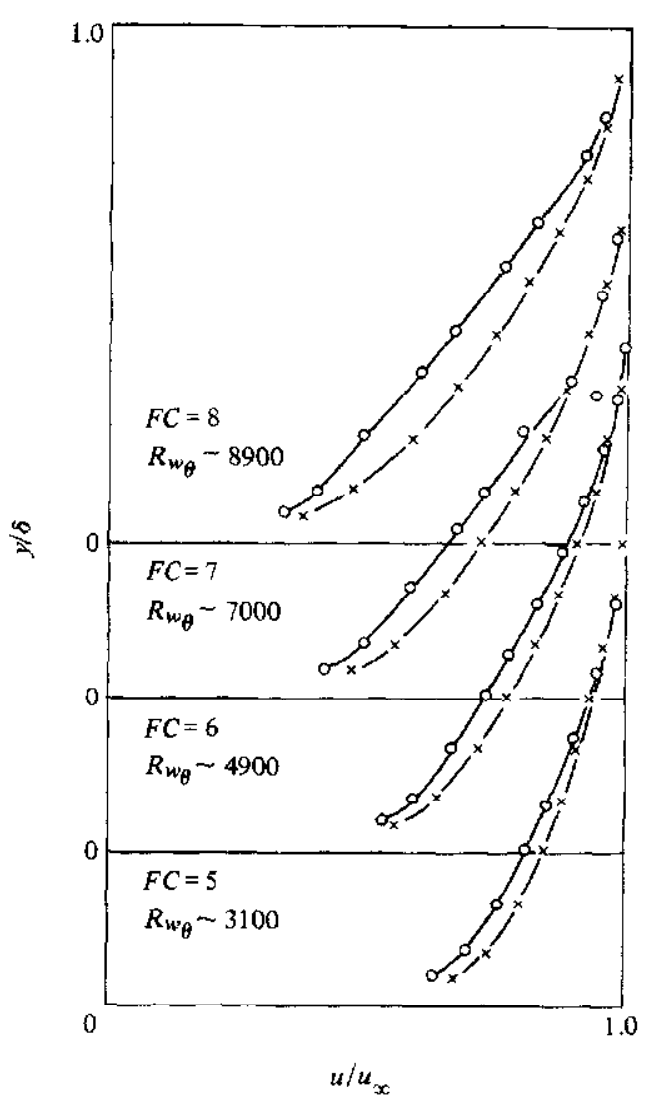

FIG. 3(b). Velocity profiles for $M=2.5: \times$, equation (7); $O$, experimental results.

the higher injection rates. This is clear indication that the strength of the wake function due to the transpiration does vary with Mach number. As a matter of fact, Figs. 3(a)-(c) show that even the wake profile for the unblown results depends strongly on Mach number as pointed out by Squire [3]. Despite that values of $\pi$ and $\pi$ identical to those in ref. [6] have been opted for in this work. Indeed, due to the uncertainties in the experimental results, it becomes very difficult to draw firm conclusions about the actual values of $A, \pi$ and $\tilde{\pi}$. Hence, it seemed sensible to assume that the expressions derived for parameters $A, \pi$ and $\tilde{\pi}$ for the incompressible case would still hold for the compressible case.

\section{FINAL REMARKS}

In this work, a new expression is introduced for the evaluation of the skin-friction for compressible turbulent bound ary layers with blowing. This expression gives much more consistent results than the momentum-integral equation and excels for its simplicity. The difficulty with the present formulation is that, in defining $\tilde{\pi}$, one is also defining both the skin-friction equation and the defect layer solution. Thus, $\tilde{\pi}$ has to be carefully determined so that both expressions provide good results. Unfortunately, the scarcity of experimental results makes this determination impossible to accomplish at this stage and so it was here decided to use values of $\tilde{\pi}$ taken from the incompressible case. This has not provided good results for the defect layer solution. Future work may even show that such a compromise in the determination of $\tilde{\pi}$ cannot be achieved and that some modifications are needed in the theory. However, this author is quite confident that, as more experimental results become available, expressions similar to equations (7) and (8) will be developed which will give good results.

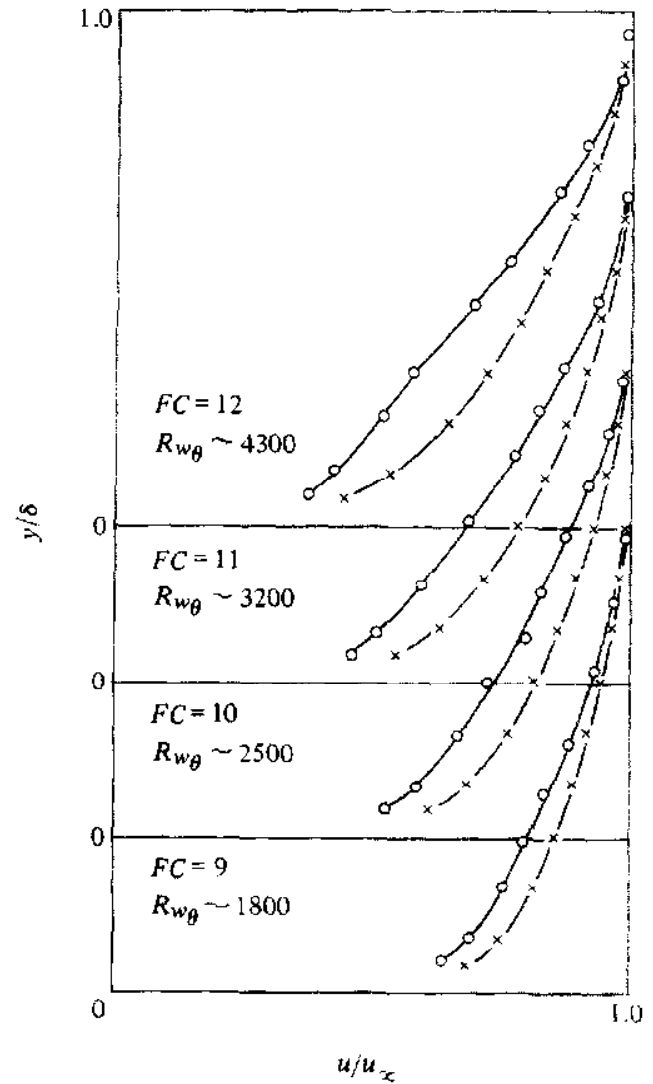

FIG. 3(c). Velocity profiles for $M=3.6: \times$, equation (7): $O$, experimental results.

Acknowledgements-The author is grateful to $\mathrm{Dr} \mathrm{L} . \mathrm{C}$. Squire for his advice and support during the course of this research. Many ideas contained in this paper are his. The work has been financially supported by the Conselho Nacional de Desenvolvimento Cientifico e TecnologicoCNPq (Brazilian National Research Council).

\section{REFERENCES}

1. L. C. Squire, A law of the wall for compressible turbulent boundary layers with air injection, J. Fluid Mech. 37,449456 (1969).

2. L. C. Squire, Further experimental investigations of compressible turbulent boundary layers with air injection, ARC R\&M No. 3627 (1970).

3. L. C. Squire, Eddy viscosity distributions in compressible turbulent boundary layers with injection, Aero $Q$. XXIII, 169-182 (1971).

4. L. C. Squire and V. K. Verma, The calculation of compressible turbulent boundary layers with fluid injection. ARC CP No. 1265 (1973).

5. L. O. F. Jeromin, An experimental investigation of the compressible turbulent boundary layer with air injection, ARC R\&M No. 3526 (1968).

6. A. P. Silva Freire, An asymptotic solution for transpired incompressible turbulent boundary layers, Int. J. Heat Mass Transfer 31, 1011-1021 (1988).

7. E. R. Van Driest, Turbulent boundary layer in compressible flow, J. Aero. Sci. 18, 145-160 (1951).

8. G. Maise and H. McDonald, Mixing length and kinematic eddy viscosity in a compressible boundary layer, AIAAJ. 6, 73-80 (1968). 